\title{
132 \\ Adaptive Hypermedia: Systems, Efficient Techniques and Implementation on the World Wide Web
}

\author{
Peter Brusilovsky \\ School of Computer Science \\ Carnegie Mellon University \\ Pittsburgh 15213, USA \\ plb@cs.cmu.edu
}

\begin{abstract}
The aim of this 3-hour tutorial is to introduce the participants to adaptive hypertext and hypermedia, a new area of research in human-computer interaction, to present a number of recent efficient adaptive hypermedia technologies on a level of detail which will enable the use of these technologies in the attendees' own systems, and demonstrate the implementation of adaptive hypermedia on the WWW. The tutorial is oriented to an intermediate level audience: $\mathrm{HCI}$ researchers and practitioners interested in designing advanced hypermedia applications and advanced WWW applications, as well as researchers who work in, or who wish to learn more about, the field of adaptive and intelligent interfaces.
\end{abstract}

KEYWORDS adaptive hypermedia, navigation, WWW, electronic textbooks, user modeling

\section{INTRODUCTION}

Adaptive hypermedia $(\mathrm{AH})$ is a new direction of research within the area of adaptive and user modelbased interfaces. Adaptive hypermedia systems build a model of the individual user and apply it for adaptation to that user, for example, to adapt the content of a hypermedia page to the user's knowledge and goals, or to suggest the most relevant links to follow. $\mathrm{AH}$ systems are used now in several application areas where the hyperspace is reasonably large and where a hypermedia application is expected to be used by individuals with different goals, knowledge and backgrounds.

The goal of the tutorial is to present a practical introduction into adaptive hypermedia for an HCI- oriented audience. This introduction will cover the following issues: a brief review of adaptive hypermedia ideas and existing systems (including Web-based systems), a detailed description of several efficient and easy-to-implement adaptation techniques, and a step-by-step instruction in "how to implement your own adaptive hypermedia system on the WWW".

The first and second issues covered by the tutorial will be most interesting for $\mathrm{HCI}$ researchers who want to learn about adaptive hypermedia, adaptive interfaces, adaptive systems, and user modeling. The second and third issues will be most interesting for the practitioners who can benefit from making their Webbased systems adaptive. To support the practical level of presentation in the third part of the tutorial, some 
minimal prerequisite knowledge about WWW, HTML, and Common Gateway Interface is desirable.

\section{OUTLINE OF THE CONTENT}

1. Introduction: what is adaptive hypermedia $(\mathrm{AH})$ and why it can be useful.

2. Where AH systems can be useful: review of existing application areas: educational hypermedia, information retrieval, on-line help and documentation systems, institutional hypermedia, personal information spaces

3. Adapting to what? Review of the most important features of the user which can be represented in the user model and used by adaptation techniques: knowledge on the subject, goals, background and experience, preferences.

4. What can be adapted in adaptive hypermedia. Introduction to general technologies of adaptation in $\mathrm{AH}$ : content adaptation, direct guidance, adaptive sorting of links, adaptive hiding of links, adaptive annotation of links, adaptive construction of hypermedia maps.

5. Methods and techniques of adaptation in $\mathrm{AH}$ systems. A review of existing techniques (about 20 existing techniques will be mentioned). Special attention will be paid to a detailed presentation of several selected techniques which proved to be efficient and can be relatively easy implemented: conditional explanations technique for content adaptation, adaptive annotation according the level of student knowledge, adaptive sorting of links according to their relevance, adaptive hiding of links to nodes "not-ready to be learned".

6. The role of user and the role of system in hypermedia adaptation. A review of different roles of user and system in the process of adaptation.

7. Efficacy of adaptive hypermedia. A brief review of known experimental studies of adaptive hypermedia. Experiments with content adaptation, adaptive sorting of links in IR hypermedia, adaptive annotation of links in educational hypermedia.

8. Lessons learned: a discussion on some requirements for creating effective adaptation techniques: transparency of adaptation, stepwise adaptation.

9. Implementation of $\mathrm{AH}$ techniques in Web based hypermedia systems. Examples. Implementation of adaptive annotation, hiding and sorting of links. Developing adaptive hypermedia with CGI scripts.
Developing adaptive hypermedia MIT CL-HTTP second generation CL-HTTP server.

\section{INSTRUCTOR}

Peter Brusilovsky received his Ph.D. in Computer Science from the Moscow State University, 1987. His main research interests are Intelligent Tutoring Systems and Shells, Student and User Modeling, Adaptive Interfaces, and Adaptive Hypermedia Systems. He is an author of about 100 papers, editor of several conference proceedings, on the editorial panel for several journals, and member of program committees for a number of international and local conferences. In particular he is an author of recent review papers including reviews on adaptive hypermedia (Brusilovsky, 1996a; Brusilovsky, 1996b). He is an editor of several hypermedia-related books and a special issue of User Modeling and UserAdapted Interaction Journal (Kluwer) on adaptive hypermedia. He has been presenting his work at many international conferences, serving also as an invited speaker at several conferences including AI-ED'95. He has experience in presenting tutorials at several local and international conferences. The current adaptive hypermedia tutorial is an update of a popular tutorial presented at ED-MEDIA'96 conference in Boston.

\section{REFERENCES}

Brusilovsky, P. (1996a) Adaptive hypermedia, an attempt to analyze and generalize. In Multimedia, Hypermedia, and Virtual Reality, Lecture Notes in Computer Science, Vol. 1077, P. Brusilovsky, P. Kommers and N. Streitz (eds), Springer-Verlag, Berlin. pp. 288-304.

Brusilovsky, P. (1996b) Methods and techniques of adaptive hypermedia. User Modeling and UserAdapted Interaction 6 (2-3), 87-129. 